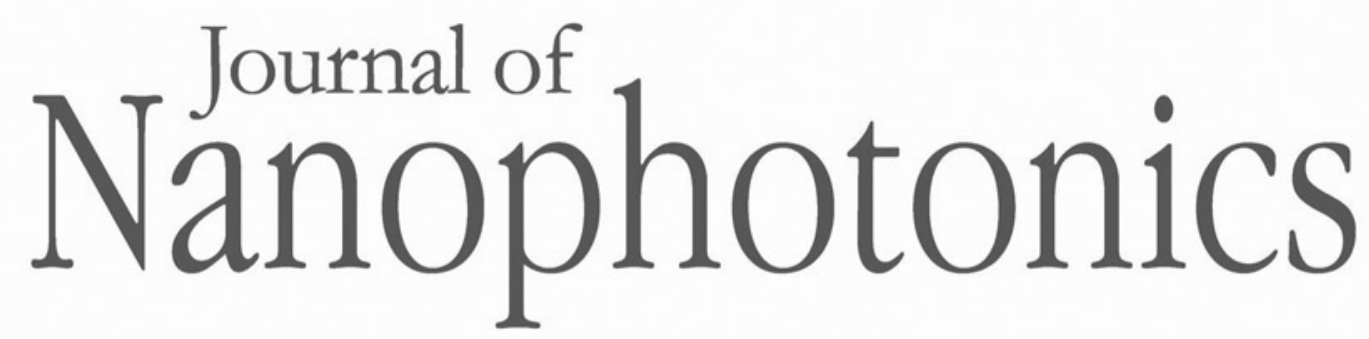

\title{
Enhancing the light extraction of AlGaN-based ultraviolet light- emitting diodes in the nanoscale
}

\author{
Yanan Guo \\ Jianchang Yan \\ Yun Zhang \\ Junxi Wang \\ Jinmin $\mathrm{Li}$
}




\title{
Enhancing the light extraction of AlGaN-based ultraviolet light-emitting diodes in the nanoscale
}

\author{
Yanan Guo, ${ }^{\text {a,b,c,d, } \dagger}$ Jianchang Yan, ${ }^{\text {a,b,c,d, },, \dagger}$ Yun Zhang, ${ }^{\text {a,b,c,d }}$

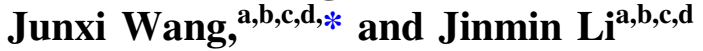 \\ ${ }^{a}$ Chinese Academy of Sciences, Institute of Semiconductors, Research and Development Center \\ for Solid State Lighting, Haidian District, Beijing, China \\ ${ }^{b}$ University of Chinese Academy of Sciences, Shijingshan District, Beijing, China \\ ${ }^{\mathrm{c}}$ Beijing Engineering Research Center for the Third-Generation Semiconductor Materials and \\ Application, Haidian District, Beijing, China \\ ${ }^{\mathrm{d} S}$ State Key Laboratory of Solid State Lighting, Haidian District, Beijing, China
}

\begin{abstract}
AlGaN-based ultraviolet light-emitting diodes (UV LEDs) are promising nextgeneration UV sources for a wide variety of applications. The state-of-the-art AlGaN-based UV LEDs exhibit much lower output power and external quantum efficiency than highly commercialized GaN visible LEDs. One key issue for UV LEDs is the poor light-extraction efficiency. We have reviewed the recent progress in the light extraction approaches for AlGaNbased UV LEDs, including the highly reflective techniques, and the surface/interface modification for total internal reflection mitigating. Moreover, AlGaN-based UV LEDs in the nanoscale structures, such as nanopillar, nanorod, and nanowire structures, are also discussed. (c) 2018 Society of Photo-Optical Instrumentation Engineers (SPIE) [DOI: 10.1117/1.JNP.12.043510]
\end{abstract}

Keywords: AlGaN; ultraviolet light-emitting diodes; light-extraction efficiency.

Paper 18071SSV received Apr. 15, 2018; accepted for publication Jun. 14, 2018; published online Jul. 6, 2018.

\section{Introduction}

AlGaN-based ultraviolet light-emitting diodes (UV LEDs) have emerged as the most promising UV sources to replace the traditional mercury lamps, due to their advantages such as compact size, low operation voltage, long lifetime, and environmental friendliness. Since the first AlGaN/ GaN quantum well $\mathrm{p}-\mathrm{n}$ diode with band-to-band recombination emission wavelength at $353.6 \mathrm{~nm}$ (shorter than the GaN bandgap wavelength) reported in $1998,{ }^{1}$ many research groups have made great efforts to develop AlGaN-based UV LEDs with shorter wavelength and higher output power; several companies have started to commercialize UV LED devices. ${ }^{2-5}$ Recently, Takano et al. ${ }^{6}$ claimed to obtain deep-UV (DUV) LEDs with a maximum external quantum efficiency (EQE) of $20.3 \%$ at $275 \mathrm{~nm}$. Inoue et al. ${ }^{7}$ reported a $265-\mathrm{nm}$ single-chip LED with a record continuous-wave output power in excess of $150 \mathrm{~mW}$ (corresponding EQE: $3.9 \%$ ) for an injection current of $850 \mathrm{~mA}$. However, the typical EQE of DUV LEDs is still in the single-digit percentage range and the output power in a couple of milliwatts, depending on the wavelength. These values are much lower than that obtained in highly commercialized GaN visible LEDs.

The low light-extraction efficiency (LEE) of AlGaN-based UV LEDs is one major bottleneck limiting the EQE. Due to the insufficient hole concentration in Al-rich p-AlGaN layer, AlGaNbased UV LEDs generally use p-GaN layer on the top of the structure to achieve hole accumulation $^{8-11}$ and ohmic contact. But the p-GaN layer would strongly absorb UV light. ${ }^{12}$ Such optical loss in the p-side can be drastically reduced when the p-GaN layer is thinned, ${ }^{13}$ partially removed, ${ }^{14,15}$ or replaced with a transparent $\mathrm{AlGaN}$ or AlGaN/AlGaN short period

\footnotetext{
*Address all correspondence to: Jianchang Yan, E-mail: yanjc@semi.ac.cn; Junxi Wang, E-mail: jxwang@semi.ac.cn

${ }^{\dagger}$ Yanan Guo and Jianchang Yan contributed equally to this work.
} 
superlattices (SPSLs) associated with highly reflective metallization stack. ${ }^{16,17}$ Furthermore, serious total internal reflection (TIR) occurs at the epitaxial layers/substrate interface and substrate/air interface because of the large difference in refraction indices. A large amount of photons are trapped inside the LED structure and finally absorbed after multiple internal reflections. Disturbing the TIR at the interfaces would be beneficial to achieve high-efficient UV LEDs. In addition, the intrinsic anisotropic optical polarization properties of AlGaN material also affect the LEE significantly. In $c$-plane AlGaN-active region, light can be emitted as either transverseelectric (TE, electrical field $\vec{E} \perp c$-axis) polarized mode or transverse-magnetic (TM, $\vec{E} \| c$-axis) polarized mode, and the predominant emission mode depends on the topmost valence subbands. The TE-polarized light mainly propagates in the vertical direction, whereas the TM-polarized light mainly propagates in the lateral direction and suffers severer TIR than the TE-polarized light. Ryu's simulation indicated that LEE of TM mode is more than 10 times smaller than that of TE mode in flip-chip DUV LEDs. ${ }^{12}$ Thus, it is necessary to develop approaches to enhance the LEE of TM-mode ${ }^{18-21}$ or promote the emission intensity of TEmode relative to TM-mode by adjusting the order of valence subbands in AlGaN, for example, adopting ( $\mathrm{Al}) \mathrm{GaN}$-delta quantum wells,${ }^{22,23} \mathrm{GaN} / \mathrm{AlN}$ quantum dot heterostructure, ${ }^{24}$ and asymmetric ultrathin $(\mathrm{GaN})_{m} /(\mathrm{AlN})_{n}(m \leq 2)$ superlattice (SPSL). ${ }^{25}$ Table 1 summarizes the current prevailing techniques for UV light extraction developed by various groups.

In this paper, we focus on the recent progress in the light extraction approaches of $\mathrm{AlGaN}$ based UV LEDs, including the highly reflective techniques, and the surface/interface modification for TIR mitigating. Moreover, UV LEDs in the nanoscale, such as nanopillar, nanorod, and nanowire structures, are also discussed.

\section{Highly Reflective Techniques for UV Light Emitters}

\subsection{Highly Reflective Electrodes}

AlGaN-based UV LEDs usually adopt flip-chip configuration in consideration of the p-GaN light-absorption issue and the thermal management. If a semi- or transparent p-layer and highly reflective structures are introduced on the top side, the upward emission light and the reflected downward light can be then reflected back to the substrate side. In consequence, the escaping probability of photons through the substrate side would be improved.

Inazu et al. ${ }^{14}$ presented a 1.55 -fold increase in LEE of a sub-300-nm DUV LED using aluminum reflective electrodes deposited to cover both $\mathrm{n}$ - and p-type mesh contact electrodes. Meanwhile, the $\mathrm{p}-\mathrm{GaN}$ layer was partially removed to increase the reflective area. Fayisa et al. ${ }^{15}$ fabricated microring array 276-nm LEDs having a p-GaN-removed inner circle of the microrings to reduce the absorption loss in the $\mathrm{p}-\mathrm{GaN}$ contact layer, together with $\mathrm{MgF}_{2} / \mathrm{Al}$ omnidirectional reflectors on the $\mathrm{p}-\mathrm{GaN}$ etched circle region to redirect the DUV photons impinging on them toward the substrate side. The microring array DUV LED showed remarkably higher light output power (LOP) by $70 \%$ than the reference.

To fundamentally avoid the p-GaN absorption problem, Shatalov et al. ${ }^{17,34}$ presented a normal incidence optical transmission at $275 \mathrm{~nm}$ increasing from 5\% in DUV LED with p-GaN contact layer to above $60 \%$ in that with transparent p-SPSL. Correspondingly, the absorption coefficient of the p-layer is dramatically reduced to below $1000 \mathrm{~cm}^{-1}$. However, the vertical conductivity of p-SPSL and contact stability then become the crucial issues and need further optimization. Hirayama et al. ${ }^{16,26,35}$ demonstrated $140 \%$ to $180 \%$ improvement in EQE of the 287/279-nm UV LEDs and 60\% increase in the electroluminescence (EL) intensity of the 260-nm DUV LED, ${ }^{36}$ when using transparent p-AlGaN contact layer and Ni/Al p-type electrode to replace $\mathrm{Ni} / \mathrm{Au}$ electrode. Yet again, this LED structure without $\mathrm{p}-\mathrm{GaN}$ contact layer also suffers from increased forward voltage due to higher p-type contact resistance than that in conventional LEDs. Recently, Zheng et al. ${ }^{37}$ proposed a multidimensional Mg-doped SL to decrease the hole potential barrier along the $c$-axis and enhance the vertical hole conductivity, which is ascribed to the stronger $\mathrm{p}_{\mathrm{z}}$ hybridization between $\mathrm{Mg}$ and $\mathrm{N}$. The grown p-type three-dimensional $\mathrm{Al}_{0.63} \mathrm{Ga}_{0.37} \mathrm{~N} / \mathrm{Al}_{0.51} \mathrm{Ga}_{0.49} \mathrm{~N}$ SLs have a hole concentration reaching a high value of $3.5 \times$ $10^{18} \mathrm{~cm}^{-3}$ and a resistivity as low as $0.7 \Omega \mathrm{cm}$ at room temperature (RT), exhibiting 10 times 
Guo et al.: Enhancing the light extraction of AIGaN-based ultraviolet. . .

Table 1 Current prevailing techniques for UV light extraction.

\begin{tabular}{|c|c|c|c|c|}
\hline Group & Year & Structures and techniques & $\begin{array}{l}\text { Wavelength } \\
\qquad(\mathrm{nm})\end{array}$ & EQE (\%) \\
\hline \multirow[t]{4}{*}{ RIKEN } & 2013 & $\begin{array}{l}\text { Transparent } p \text {-AIGaN contact layer and } \\
\text { highly reflective } p \text {-type electrode }{ }^{16}\end{array}$ & 287 & $5.5 @ 35 \mathrm{~A} / \mathrm{cm}^{2}$ \\
\hline & 2017 & $\begin{array}{l}\text { Transparent } \mathrm{p}-\mathrm{AIGaN} \text { contact layer, } \mathrm{Rh} \\
\text { mirror electrode, patterned sapphire } \\
\text { substrate, and encapsulation resin }{ }^{6}\end{array}$ & 275 & $20.3 @ 20 \mathrm{~mA}$ \\
\hline & 2018 & Highly reflective Ni/Al p-type electrodes ${ }^{26}$ & 279 & $9 @ 20 \mathrm{~mA}$ \\
\hline & 2018 & $\begin{array}{l}\text { HR PhC on p-AIGaN contact layer and } \\
\text { reflective Ni/Mg contact layer }{ }^{27}\end{array}$ & 283 & $\begin{array}{c}10 @ 3 \mathrm{~mA} \\
>8.5 @ 20 \mathrm{~mA}\end{array}$ \\
\hline $\begin{array}{l}\text { Sensor Electronic } \\
\text { Technology, Inc. }\end{array}$ & 2012, 2015 & $\begin{array}{l}\text { Transparent } p \text {-cladding and contact } \\
\text { layers, UV reflective ohmic contact, and } \\
\text { chip encapsulation }{ }^{17,28}\end{array}$ & 278 & $10.4 @ 20 \mathrm{~mA}$ \\
\hline $\begin{array}{l}\text { National Institute } \\
\text { of Information and } \\
\text { Communications } \\
\text { Technology }\end{array}$ & 2015,2017 & $\begin{array}{l}\text { Large-area nanoimprinted AIN } \\
\text { nanophotonic light-extraction structure }\end{array}$ & 265 & $\begin{array}{l}4.5 @ 150 \mathrm{~mA} \\
3.9 @ 850 \mathrm{~mA}\end{array}$ \\
\hline \multirow[t]{3}{*}{ UV Craftory Co. } & 2011 & $\begin{array}{l}\mathrm{P} \text { - and } \mathrm{n} \text {-mesh contact electrodes } \\
\text { covered by aluminum reflective } \\
\text { electrodes }^{14}\end{array}$ & 288 & $5.4 @ 20 \mathrm{~mA}$ \\
\hline & 2013 & $\begin{array}{l}\text { Flip-chip bonding structure with resistant } \\
\text { resin encapsulation }\end{array}$ & 280 to 310 & $10(\max .14 .2)$ \\
\hline & & & 260 & 5 \\
\hline \multirow[t]{3}{*}{ Crystal IS } & 2013 & $\begin{array}{l}\text { Die (AIN substrate) thinning and } \\
\text { encapsulation }{ }^{31,32}\end{array}$ & 271 & $5.8 @ 60 \mathrm{~mA}$ \\
\hline & & & 266 & $5.5 @ 50 \mathrm{~mA}$ \\
\hline & & & 278 & $7 @ 50 \mathrm{~mA}$ \\
\hline \multirow[t]{2}{*}{ Nichia Corp. } & 2016 & With sapphire lens using direct bonding & 255 & $4.56 @ 100 \mathrm{~mA}$ \\
\hline & & & 280 & $10.1 @ 1 \mathrm{~A}$ \\
\hline \multirow[t]{2}{*}{$\begin{array}{l}\text { Pohang University } \\
\text { of Science and } \\
\text { Technology }\end{array}$} & 2016 & $\begin{array}{l}\text { Multiple mesa stripes or truncated } \\
\text { cone-shaped mesa arrays with inclined } \\
\text { sidewalls coated by a } \mathrm{MgF}_{2} / \mathrm{Al} \\
\text { omnidirectional mirror }^{20,33}\end{array}$ & 280 & - \\
\hline & 2017 & $\begin{array}{l}5 \times 5 \text { array microring LED with a } \mathrm{p}-\mathrm{GaN}- \\
\text { removed inner circle and inclined } \\
\text { sidewalls coated by } \mathrm{MgF}_{2} / \mathrm{Al} \\
\text { omnidirectional reflectors }^{15}\end{array}$ & 276 & - \\
\hline $\begin{array}{l}\text { Institute of } \\
\text { Semiconductors, } \\
\text { Chinese Academy } \\
\text { of Sciences }\end{array}$ & 2013 & Nano-patterned sapphire substrate ${ }^{11}$ & 282 & $3.45 @ 20 \mathrm{~mA}$ \\
\hline
\end{tabular}

improvement in conductivity compared with that of conventional SLs. Moreover, the resistivity is weakly dependent on temperature. The advances of multidimensional SLs provide a promising solution to reduce the forward voltage of UV LEDs.

Vertical emission can be obtained from the standing wave formed by lateral Bragg resonance in a two-dimensional photonic crystal $(\mathrm{PhC})$ near the quantum-well emission layer. ${ }^{38}$ Kashima et al. ${ }^{27}$ introduced a highly reflective $\mathrm{PhC}(\mathrm{HR}-\mathrm{PhC})$ into the surface of the p-AlGaN contact layer to induce the vertical light-propagation. According to their results, the effective reflectance of the HR-PhC p-AlGaN contact layer with the $\mathrm{Ni} / \mathrm{Mg}$ electrode exceeded $90 \%$. The EQE of 
a conventional DUV LED with emission around $283 \mathrm{~nm}$ was increased from $4.8 \%$ to $10 \%$ by using this highly reflective structure.

\subsection{Sidewall Reflection Enhancement Structure}

Highly reflective structure at the slant mesa sidewalls can efficiently deflect the photons guided laterally along the $c$-plane into the vertical direction, which seems a tailored technique for the extraction of TM-polarized light. ${ }^{39}$ Lee et al. ${ }^{20}$ reported sidewall-emission-enhanced DUV LEDs with active mesa stripes or arrays of truncated cone-shaped active mesas ${ }^{33}$ coated with $\mathrm{MgF}_{2} / \mathrm{Al}$ reflectors to effectively extract the in-plane TM-polarized emission.

We reported a sidewall reflection enhancement method for AlGaN-based 278-nm LEDs, consisting of mesa microarrays in a hexagonal shape and on-chip highly reflective metalpads for UV light, ${ }^{21}$ as shown in Fig. 1 . The total device area was $1 \mathrm{~mm}^{2}$. Each independent micromesa had a side length of $30 \mu \mathrm{m}$ and a trapezoidal cross-sectional shape with a slant angle of $\sim 45 \mathrm{deg}$ after the inductively coupled plasma dry etching. A Ti/Al/Ti/Au metal stack and a $\mathrm{Ni} / \mathrm{Au}$ metal stack were deposited on the exposed $\mathrm{n}-\mathrm{AlGaN}$ layer and $\mathrm{p}-\mathrm{GaN}$ layer to form ohmic contacts, respectively. After the chip passivation with $\mathrm{SiO}_{2}$, chip-level metal-pads were deposited as anode/cathode electrodes and bond pads as well for the subsequent flip-chip process. Here, we compared the influence of two kinds of metal-pads on the LOP of AlGaN UV LEDs. Cr/Al/Ti/Au system is widely used in the standard 450-nm blue LED fabrication, but $\mathrm{Cr}$ metal is not a good UV reflector. In our new method, aluminum-based metal-pads, i.e., $\mathrm{Al} / \mathrm{Ti} / \mathrm{Au}$, were evaporated, which not only acted as electrodes and bond pads but also covered the trapezoidal mesa sidewall to accommodate the light propagation path. Then, the separated LED chips were flip-chip bonded on silicon submounts with gold bumps for further device testing. It should be noted that the reflectivity of the on-chip metal-pads maintains consistently because of no high-temperature treatment involved.

The average LOP of the encapsulated LEDs with highly reflective metal-pads was increased by $30.1 \%$, compared to that with traditional metal-pads. The improvement is attributed to the less absorption and the more efficient reflection of in-plane guided light arriving at the mesa sidewalls. ${ }^{39}$ The LOP can be further increased via reducing the sidewall length of the mesa due to the larger sidewall area for DUV reflection. In addition, there were no significant differences between these two kinds of chips in the electrical properties and the yield, revealing a quite good compatibility of Al/Ti/Au metal-pads in the UV LED fabrication process.

Figure 2 shows the performance of one LED using the sidewall reflection method as a function of the injected CW current. At $100 \mathrm{~mA}$, the LOP reaches $6.8 \mathrm{~mW}$ and the forward voltage is about $7 \mathrm{~V}$. When driven to $500 \mathrm{~mA}$, the LED presents a saturation LOP of around $25 \mathrm{~mW}$ due to the increased Auger recombination under high current density and heat. The device was stressed at $100 \mathrm{~mA} \mathrm{CW}$ for $>6000 \mathrm{~h}$ at RT. The LOP decay shows the L80 $(80 \%$ of the initial power retention) operation lifetime of $\sim 5000 \mathrm{~h}$ and extrapolated L50 lifetime to $>10,000 \mathrm{~h}$.

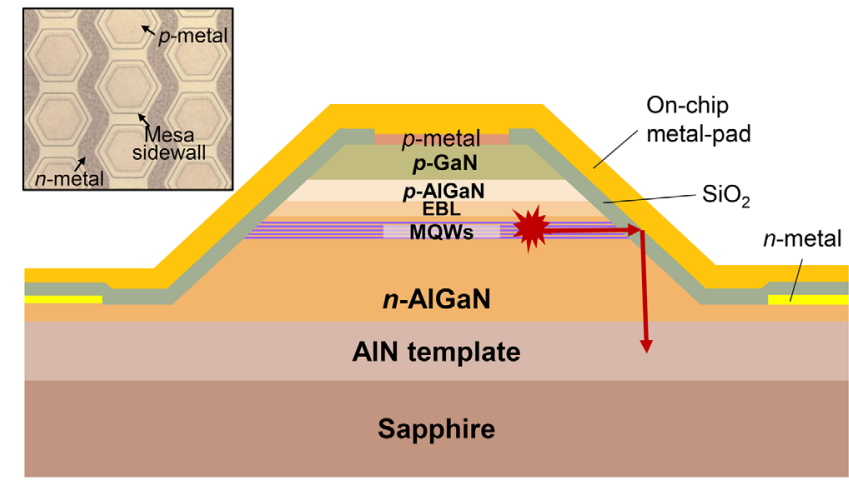

Fig. 1 The schematic structure of AIGaN-based UV LEDs using a sidewall reflection method. Inset: The top-view of the LED chip before the deposition of on-chip metal-pads. 

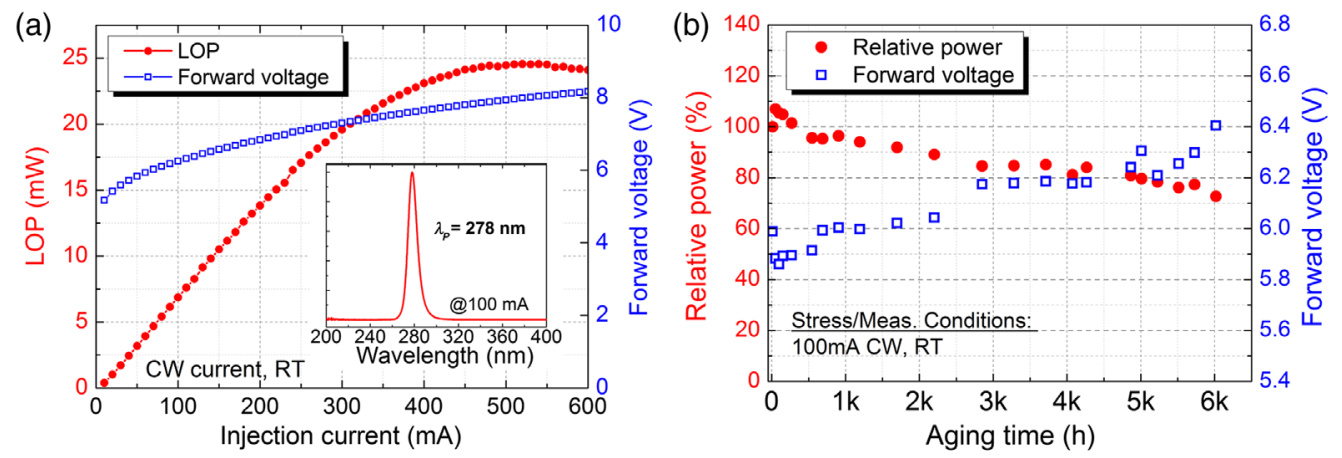

Fig. 2 (a) The typical LOP- and forward voltage-injected CW current characteristics. Inset: the EL spectrum at $100 \mathrm{~mA}$; (b) the reliability test of an encapsulated DUV LED with the sidewall reflection structure at $100-\mathrm{mA} \mathrm{CW}$ and RT.

\section{Surface/Interface Modification Techniques for UV LEDs}

\subsection{Substrate Surface Roughening}

Substrate surface roughening is meant to provide numerous scattering events at the substrate/ ambient interfaces for trapped photons to escape from the LED structure. Khizar et al. ${ }^{40}$ reported on the fabrication of AlGaN-based 280-nm LEDs with monolithic integrated microlens array on the backside of sapphire substrates and proved a 55\% enhancement in LOP at $20 \mathrm{~mA}$ compared with the same LED without miroclens. Pernot et al. ${ }^{41}$ demonstrated a $50 \%$ increase in on-wafer output power of 270-nm LEDs by fabricating a moth-eye structure on the sapphire substrate. Inoue et al. ${ }^{29}$ designed hybrid structure nanostructure of PhCs and subwavelength nanostructures on HVPE-AlN substrates. A maximum light extraction enhancement of $196 \%$ was obtained in 265-nm LEDs with the hybrid structure in comparison to that of a flat surface device. To enable high-output and high-volume fabrication, they further presented 265-nm DUV LEDs with largearea nanoimprinted AlN nanophotonic structures, ${ }^{7}$ which showed wider near-field emitting areas, stronger far-field extracted light intensities, and an approximately 20 -fold increase in LOP compared with a conventional flat-surface LED.

\subsection{Substrate Sidewall Roughening}

As mentioned above, the TM-polarized light propagates mainly in the lateral direction and is extracted from the device sidewalls. Hence, it is also important to pay attention to the sidewall TIR mitigation such as roughening the device sidewalls, especially the substrate sidewalls that have lager area than the epitaxial layer sidewalls.

Substrate sidewall roughening can be realized by ultrashort pulse laser stealth dicing during the chip-separation process and has been proven to be helpful to enhance the LEE in visible and UV LEDs. ${ }^{42-45}$ Lee at al. ${ }^{45}$ demonstrated that thick sapphire substrates with roughened sidewalls are critical for TM-polarized photons from the AlGaN quantum wells to escape in lateral direction before they are absorbed by $\mathrm{p}-\mathrm{GaN}$ and metal electrodes. We investigated the effect of sapphire substrate's sidewall roughening on the light extraction behavior of AlGaN-based 275-nm UV LEDs by finite-difference time-domain (FDTD) simulation and experimental output power measurement. ${ }^{46}$

Figure 3(a) shows the FDTD computational domain of the UV LED with a roughened sapphire substrate reference sidewall. For a given TE- or TM-mode dipole with a distance $d$ from the reference sidewall, its light extraction is enhanced only when the roughening position $z$ is within a specific region (not shown here), which is defined as the effective roughening region. As shown in Fig. 3(b), the effective roughening region is between the substrate's backside and the lower boundary $\sim d \times \tan (\theta c)$, where $\theta c$ is the TIR critical angle. Roughening outside the effective roughening region will reduce the sidewall LEE due to the undesirable inward photon scattering at the substrate sidewall. The extracted light power of various dipoles with different positions was simulated individually and then combined to obtain the overall light extraction enhancement 
(a)

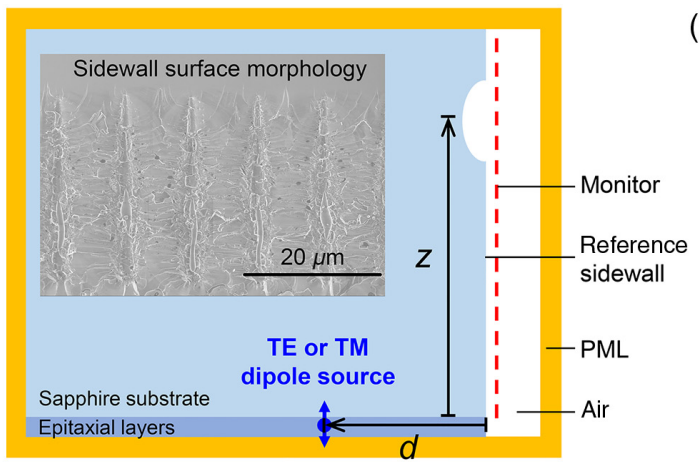

(b)

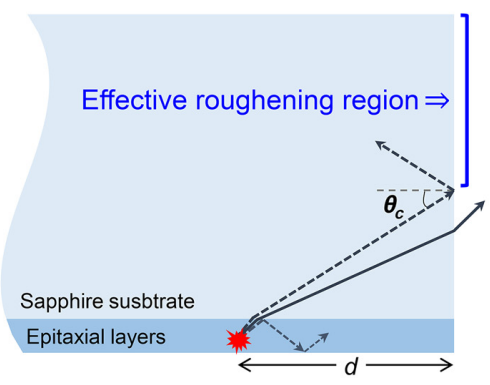

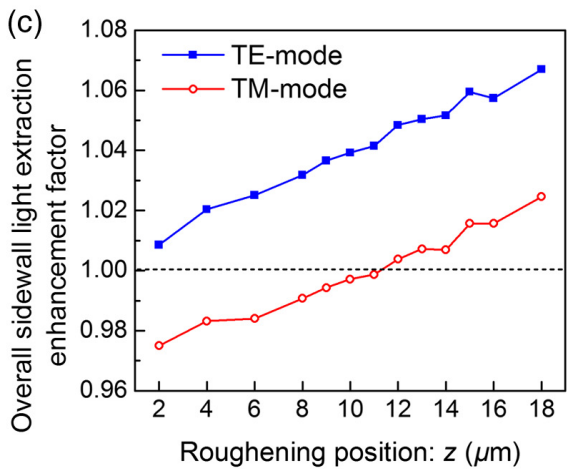

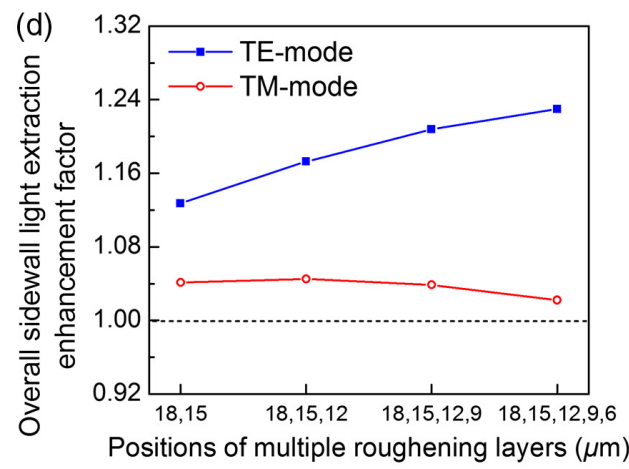

Fig. 3 (a) Schematic diagram of the FDTD computational domain of the UV LED structure with the roughened sapphire sidewall. Inset: Scanning electron microscopy (SEM) image of sidewall morphology of one roughening layer. ${ }^{46}$ (b) Schematic diagram of the effective roughening region for one dipole on the reference sidewall. The dependence of overall sidewall light extraction enhancement factor on the position of (c) a single roughening layer and (d) multiple roughening layers.

factor with respect to the roughening position. As shown in Fig. 3(c), the overall extraction efficiency of TE-mode light keeps improved regardless of the roughening position $z$ on the substrate sidewall. In contrast, the overall extraction of TM-mode light encounters a decrease when the roughening position $z$ is lower than a certain value, revealing that a roughening region for effective light extraction enhancement exists on the substrate's sidewall. Moreover, the boundary of the overall effective roughening region of TM mode is found equal to that of the TM-polarized dipole in the center of the active region. ${ }^{46} \mathrm{~A}$ simulation with multiple roughening layers in Fig. 3(d) further verified the effective roughening region assumption. Therefore, the effective roughening region of TM polarization in one LED with a side length of $L$ is deduced to from the sapphire substrate's backside to the position $\sim L / 2 \times \tan (\theta c)$.

We designed four sidewall roughening schemes for TM-mode-dominant UV LEDs. As shown in Fig. 4, the LEDs with three roughening layers have 13.2\% higher LOP at $20 \mathrm{~mA}$ than those with two roughening layers, due to the larger roughening area in the effective roughening region. However, when further applying additional roughening layers beyond the effective roughening region, the LOP decreases, which is in consistent with the simulation result. The current-voltage characteristics of these LEDs were similar, indicating that the LOP variation mainly comes from different roughening schemes. Therefore, depth-controlled substrate's sidewall roughening is beneficial to maximize the LOP of AlGaN-based UV LEDs.

\subsection{Substrate Shaping}

Modifying the geometry of the LED chip can also help outcouple the light trapping in the structure. It could be regarded as the upscaling of the surface texturing. ${ }^{47}$ Krames et al. ${ }^{48}$ fabricated truncated-inverted-pyramid AlGaInP-based LEDs sawn by a beveled dicing blade, exhibiting $40 \%$ improvement in EQE compared to the conventional devices. Lee et al. ${ }^{49}$ reported $55 \%$ light output enhancement in flip-chip GaN-based LEDs with oblique sapphire sidewalls on the bottom of the substrate using a wet etching technique. 


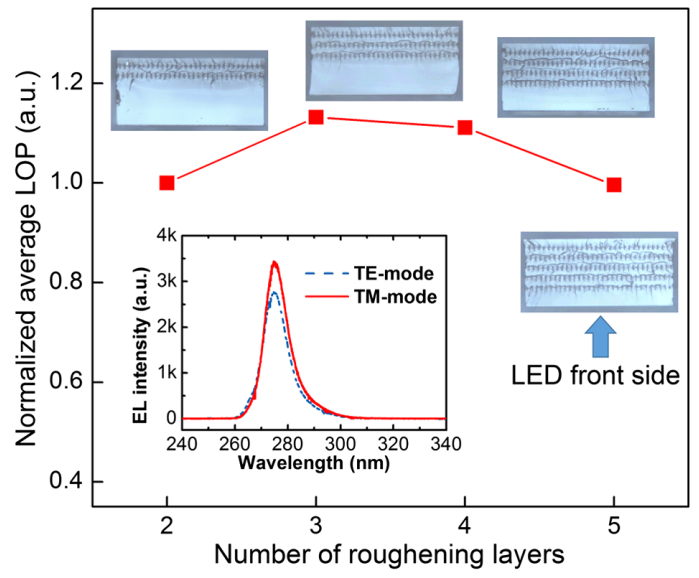

Fig. 4 The normalized average LOP of LEDs at $20 \mathrm{~mA}$. Inset: The EL spectra of the LED wafer for TE- and TM-modes at $20 \mathrm{~mA}$.

Laser micromachining has been introduced to shape the sapphire substrate, such as nanosecond laser dicing either with a laser-beam turning mirror ${ }^{50}$ or with an oblique sample stage ${ }^{51}$ to bevel the substrate sidewalls and shifted picosecond (ps) laser stealth dicing to form wavy substrate sidewalls. ${ }^{52}$ We proposed a convenient ps laser multiple scribing method to shape the sapphire substrates into an oblique sidewall geometry for enhanced LEE. ${ }^{53}$ In general, the scribing lines beneath the same street are distributed in the same vertical plane inside the substrate; thus, the LEDs have vertical sidewalls after splitting. In our study, the applied multiple scribing lines beneath the same street are intentionally aligned along an oblique plane with designed angles to guide the chip splitting direction. The LED chips are thereby shaped into an oblique sidewall geometry. Compared with the convention chip with vertical sidewalls, the 276-nm LED with two opposite sidewalls partially shaped with 60 deg inclination shows $13.8 \%$ higher LOP at $50 \mathrm{~mA}$. The FDTD simulation reveals that this oblique substrate sidewall geometry is effective for TM-polarized light extraction.

\subsection{Interface Modification}

According to the simulations ${ }^{12,21,54}$ and experimental results, a large amount of TE- and TMpolarized light encounter TIR at the interfaces among the substrate, AlN layer, n-AlGaN layer, multiple-quantum-well region, electron blocking layer, and p-AlGaN cladding layer, resulting in light confinement and absorption. A careful consideration should be given to the interface modification, or in other words, effective refraction index adjustment around the interfaces.

The AIN epitaxial lateral overgrowth on pattered substrates or templates can not only improve the crystal quality of the overgrown epitaxial layers but also form embedded air voids in the AIN layer (as shown in Fig. 5). ${ }^{11,35,55-57}$ The effective refraction index around the interface is thereby between the AlN layer and the substrates (or the templates). The light scattering at the interface reduces the TIR and increases the photons' escape opportunity. We reported AlGaN-based 282-nm LEDs grown on nanopatterned sapphire substrates (NPSS), exhibiting $98 \%$ better performance relative to those grown flat sapphire substrate. ${ }^{11}$ The internal quantum efficiency (IQE) enhancement is estimated to be $60 \%$ enhancement, so the LEE enhancement would be more than $20 \%$. Lee et al. ${ }^{56}$ fabricated DUV LEDs on NP-AlN/sapphire templates, with air surrounding the AIN nanorods. Light emitted from the multiple quantum wells can propagate vertically by passing through the embedded nanostructures and thus the TIR is avoided.

\section{Nanostructure UV LEDs}

The nanostructures, including nanorods, nanopillars, nanowires, or other structures in the nanoscale, have been found to be quite effective in improving the LEE of AlGaN-based UV LEDs. 


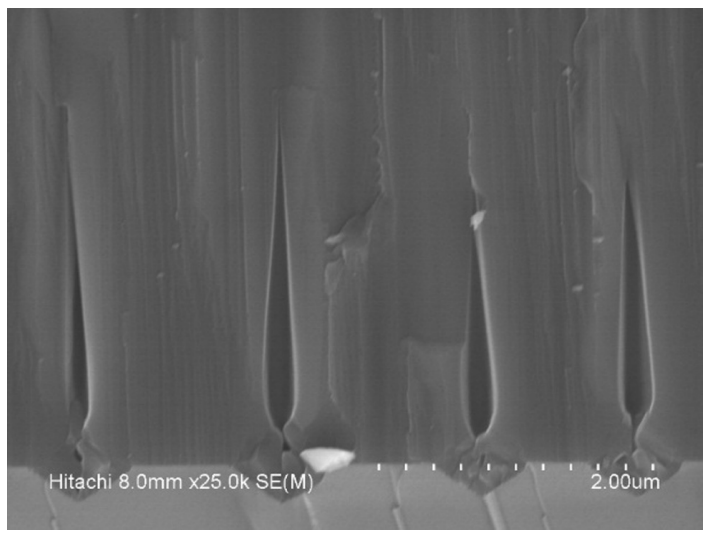

Fig. 5 A cross-sectional SEM image of overgrown AIN on NPSS.

Ryu's study showed that the LEE from the top surface can be higher than $50 \%$ and $60 \%$ for TEand TM-polarized lights, respectively, when the structural parameters of the nanorod LED are optimized for the formation of resonant modes inside the nanorod structure. ${ }^{58}$ Zhao et al. ${ }^{19}$ reported an efficient surface-emitting near-vacuum UV nanowire LED through direct electrical injection. By optimizing the nanowire diameter and spacing, the dominated light emission can be from the nanowire top surface due to the multiple light coupling and scattering process, albeit the light is TM polarized. Djavid et al.'s simulation showed that the emission of the guided modes in DUV LEDs can be inhibited and redirected into radiated modes utilizing nanowire structure. ${ }^{59,60}$ Alias et al. ${ }^{61}$ demonstrated an AlGaN nanowire LED integrated with nitride/air distributed Bragg reflector nanogratings to enhance the LEE. Compared to the control AlGaN NW-LED, the LED with the nanogratings exhibited higher LOP by a factor of $\sim 1.67$ at $50 \mathrm{~mA}$ injected current, which is attributed to the coupling of the in-plane backreflected light from the nanogratings and the out-of-plane emission. In addition, the nonvertical sidewalls of the nanogratings also facilitate the in-plane light to scatter to the out-of-plane emission.

Nanostructured AlGaN-based UV LEDs can be obtained either by the bottom-up growth or top-down etching method. Using the former bottom-up method mainly by molecular beam epitaxy, nearly defect-free $\mathrm{Al}(\mathrm{Ga}) \mathrm{N}$ nanowires/nanorods can be grown on foreign substrates with high internal quantum efficiency (IQE) ${ }^{62-64}$ Moreover, the enhanced Mg-dopant incorporation and the reduced activation energy of $\mathrm{Mg}$ dopant in nanostructures lead to significantly improved p-type doping efficiency and more efficient current injection. But it should be mentioned that the inhomogeneity of the nanostructures can cause unexpected light confinement and material lightabsorption loss.

In contrast, ordered nanostructures can be achieved via the latter etching method. ${ }^{18,65}$ We fabricated nanopillar AlGaN/GaN multiple quantum wells UV LEDs by nanosphere lithography and dry-etching. ${ }^{18}$ The simulated LEE from the top surface, bottom surface, and the sidewalls in the nanopillar LED is $2.2,1.8$, and 0.73 times of those in the planar LED, respectively. Although the LEE from the sidewalls is decreased, the total LEE is still increased from $22.5 \%$ to $33.7 \%$ due to the greatly enhanced LEE in the vertical direction. In addition, the strain is partially released in the epitaxial layers of the nanopillar LED, resulting in suppressed quantum confined stark effect and a $42 \%$ enhanced IQE at RT.

\section{Conclusions}

This study reviewed the recent progress in the light extraction approaches of AlGaN-based UV LEDs, including the highly reflective techniques and the surface/interface modification for TIR mitigating. Moreover, AlGaN-based UV LEDs in the nanoscale structures, such as nanopillar, nanorod, and nanowire structures, are also discussed. Nanostructured UV LEDs have been found to be quite effective in improving the LEE especially the LEE of TM-polarized light.

\section{Disclosures}

The authors declare no competing financial interests. 


\section{Acknowledgments}

This work was supported by the National Key R\&D Program of China (Grant Nos. 2016YFB0400803 and 2016YFB0400802), the National Natural Science Foundation of China (Grant Nos. 61527814, 61674147, and U1505253), Beijing Nova Program Z181100006218007, and Youth Innovation Promotion Association CAS 2017157.

\section{References}

1. J. Han et al., "AlGaN/GaN quantum well ultraviolet light emitting diodes," Appl. Phys. Lett. 73(12), 1688-1690 (1998).

2. M. Kneissl and J. Rass, III-Nitride Ultraviolet Emitters, Springer International Publishing, Cham (2016).

3. Z. Mi and C. Jagadish, III-Nitride Semiconductor Optoelectronics, Elsevier, Cambridge (2017).

4. J. M. Li et al., "Advances and prospects in nitrides based light-emitting diodes," J. Semicond. 37(6), 061001 (2016).

5. D. Li et al., "AlGaN photonics: recent advances in materials and ultraviolet devices," $A d v$. Opt. Photonics 10(1), 43 (2018).

6. T. Takano et al., "Deep-ultraviolet light-emitting diodes with external quantum efficiency higher than $20 \%$ at $275 \mathrm{~nm}$ achieved by improving light-extraction efficiency," Appl. Phys. Express 10(3), 031002 (2017).

7. S.-I. Inoue, N. Tamari, and M. Taniguchi, "150 mW deep-ultraviolet light-emitting diodes with large-area AIN nanophotonic light-extraction structure emitting at $265 \mathrm{~nm}$," Appl. Phys. Lett. 110(14), 141106 (2017).

8. M. S. Shur et al., "Accumulation hole layer in p-GaN/AlGaN heterostructures," Appl. Phys. Lett. 76(21), 3061-3063 (2000).

9. V. Adivarahan et al., "AlGaN single-quantum-well light-emitting diodes with emission at 285 nm," Appl. Phys. Lett. 81(19), 3666-3668 (2002).

10. H. Hirayama et al., "Marked enhancement in the efficiency of deep-ultraviolet AlGaN lightemitting diodes by using a multiquantum-barrier electron blocking layer," Appl. Phys. Express 3(3), 031002 (2010).

11. P. Dong et al., "282-nm AlGaN-based deep ultraviolet light-emitting diodes with improved performance on nano-patterned sapphire substrates," Appl. Phys. Lett. 102(24), 241113 (2013).

12. H. Y. Ryu et al., "Investigation of light extraction efficiency in AlGaN deep-ultraviolet lightemitting diodes," Appl. Phys. Express 6(6), 062101 (2013).

13. M. Akiba et al., "Growth of flat p-GaN contact layer by pulse flow method for high lightextraction AlGaN deep-UV LEDs with Al-based electrode," Phys. Status Solidi C 9(3-4), 806-809 (2012).

14. T. Inazu et al., "Improvement of light extraction efficiency for AlGaN-based deep ultraviolet light-emitting diodes," Jpn. J. Appl. Phys. 50, 122101 (2011).

15. G. B. Fayisa et al., "Enhanced light extraction efficiency of micro-ring array AlGaN deep ultraviolet light-emitting diodes," Jpn. J. Appl. Phys. 56(9), 092101 (2017).

16. N. Maeda and H. Hirayama, "Realization of high-efficiency deep-UV LEDs using transparent p-AlGaN contact layer," Phys. Status Solidi C 10(11), 1521-1524 (2013).

17. M. Shatalov et al., "AlGaN deep-ultraviolet light-emitting diodes with external quantum efficiency above 10\%," Appl. Phys. Express 5(8), 082101 (2012).

18. P. Dong et al., "Optical properties of nanopillar AlGaN/GaN MQWs for ultraviolet lightemitting diodes," Opt. Express 22(S2), A320-A327 (2014).

19. S. Zhao, M. Djavid, and Z. Mi, "Surface emitting, high efficiency near-vacuum ultraviolet light source with aluminum nitride nanowires monolithically grown on silicon," Nano Lett. 15(10), 7006-7009 (2015).

20. J. W. Lee et al., "An elegant route to overcome fundamentally-limited light extraction in AlGaN deep-ultraviolet light-emitting diodes: preferential outcoupling of strong in-plane emission," Sci. Rep. 6, 22537 (2016). 
21. Y. Guo et al., "Enhancement of light extraction on AlGaN-based deep-ultraviolet lightemitting diodes using a sidewall reflection method," in 13th China Int. Forum on Solid State Lighting: Int. Forum on Wide Bandgap Semiconductors China (SSLChina: IFWS), pp. 127-130, Beijing, China (2016).

22. J. Zhang, H. Zhao, and N. Tansu, "Large optical gain AlGaN-delta-GaN quantum wells laser active regions in mid- and deep-ultraviolet spectral regimes," Appl. Phys. Lett. 98(17), 171111 (2011).

23. C. Liu et al., " $234 \mathrm{~nm}$ and $246 \mathrm{~nm}$ AlN-Delta-GaN quantum well deep ultraviolet lightemitting diodes," Appl. Phys. Lett. 112(1), 011101 (2018).

24. J. Verma et al., "Tunnel-injection GaN quantum dot ultraviolet light-emitting diodes," Appl. Phys. Lett. 102(4), 041103 (2013).

25. W. Lin et al., "Optical isotropization of anisotropic wurtzite Al-rich AlGaN via asymmetric modulation with ultrathin $(\mathrm{GaN}) \mathrm{m} /(\mathrm{AlN}) \mathrm{n}$ superlattices," Laser Photonics Rev. 7(4), 572 579 (2013).

26. N. Maeda, M. Jo, and H. Hirayama, "Improving the efficiency of AlGaN deep-UV LEDs by using highly reflective Ni/Al p-type electrodes," Phys. Status Solidi A 215, 1700435 (2018).

27. Y. Kashima et al., "High external quantum efficiency (10\%) AlGaN-based deep-ultraviolet light-emitting diodes achieved by using highly reflective photonic crystal on p-AlGaN contact layer," Appl. Phys. Express 11(1), 012101 (2018).

28. M. Shatalov et al., "High-efficiency UV LEDs on sapphire," Proc. SPIE 9363, 93631M (2015).

29. S.-I. Inoue et al., "Light extraction enhancement of $265 \mathrm{~nm}$ deep-ultraviolet light-emitting diodes with over $90 \mathrm{~mW}$ output power via an AlN hybrid nanostructure," Appl. Phys. Lett. 106(13), 131104 (2015).

30. M. Ippommatsu et al., "Development of AlGaN DUV-LED," in Conf. on Lasers and Electro-Optics Pacific Rim (CLEO-PR), pp. 1-2, IEEE, Kyoto, Japan (2013).

31. J. R. Grandusky et al., " $270 \mathrm{~nm}$ pseudomorphic ultraviolet light-emitting diodes with over $60 \mathrm{~mW}$ continuous wave output power," Appl. Phys. Express 6(3), 032101 (2013).

32. C. G. Moe et al., "High-power pseudomorphic mid-ultraviolet light-emitting diodes with improved efficiency and lifetime," Proc. SPIE 8986, 89861V (2014).

33. J. W. Lee et al., "Arrays of truncated cone AlGaN deep-ultraviolet light-emitting diodes facilitating efficient outcoupling of in-plane emission," ACS Photonics 3(11), 20302034 (2016).

34. M. Shatalov et al., "High power AlGaN ultraviolet light emitters," Semicond. Sci. Technol. 29(8), 084007 (2014).

35. H. Hirayama et al., "Recent progress and future prospects of AlGaN-based high-efficiency deep-ultraviolet light-emitting diodes," Jpn. J. Appl. Phys. 53(10), 100209 (2014).

36. M. Jo, N. Maeda, and H. Hirayama, "Enhanced light extraction in $260 \mathrm{~nm}$ light-emitting diode with a highly transparent p-AlGaN layer," Appl. Phys. Express 9(1), 012102 (2016).

37. T. C. Zheng et al., "Improved p-type conductivity in Al-rich AlGaN using multidimensional Mg-doped superlattices," Sci. Rep. 6, 21897 (2016).

38. Y. Kurosaka et al., "Controlling vertical optical confinement in two-dimensional surfaceemitting photonic-crystal lasers by shape of air holes," Opt. Express 16(22), 18485-18494 (2008).

39. J. J. Wierer et al., "Influence of optical polarization on the improvement of light extraction efficiency from reflective scattering structures in AlGaN ultraviolet light-emitting diodes," Appl. Phys. Lett. 105(6), 061106 (2014).

40. M. Khizar et al., "Nitride deep-ultraviolet light-emitting diodes with microlens array," Appl. Phys. Lett. 86(17), 173504 (2005).

41. C. Pernot et al., "Improved efficiency of $255-280 \mathrm{~nm}$ AlGaN-based light-emitting diodes," Appl. Phys. Express 3(6), 061004 (2010).

42. J. H. Lee et al., "Enhanced extraction efficiency of InGaN-based light-emitting diodes using 100-kHz femtosecond-laser-scribing technology," IEEE Electron Device Lett. 31(3), 213215 (2010).

43. K. C. Chen et al., "Laser scribing of sapphire substrate to increase side light extraction of GaN-based light emitting diodes," J. Lightwave Technol. 29(13), 1907-1912 (2011). 
44. Y. Y. Zhang et al., "Light extraction efficiency improvement by multiple laser stealth dicing in InGaN-based blue light-emitting diodes," Opt. Express 20(6), 6808-6815 (2012).

45. K. H. Lee et al., "Light-extraction efficiency control in AlGaN-based deep-ultraviolet flipchip light-emitting diodes: a comparison to InGaN-based visible flip-chip light-emitting diodes," Opt. Express 23(16), 20340-20349 (2015).

46. Y. Guo et al., "Light extraction enhancement of AlGaN-based ultraviolet light-emitting diodes by substrate sidewall roughening," Appl. Phys. Lett. 111(1), 011102 (2017).

47. A. I. Zhmakin, "Enhancement of light extraction from light emitting diodes," Phys. Rep. 498(4-5), 189-241 (2011).

48. M. R. Krames et al., "High-power truncated-inverted-pyramid $\left(\mathrm{A}_{1 x} \mathrm{Ga}_{1-\mathrm{x}}\right)_{0.5} \mathrm{In}_{0.5} \mathrm{P} / \mathrm{GaP}$ light-emitting diodes exhibiting $>50 \%$ external quantum efficiency," Appl. Phys. Lett. 75(16), 2365-2367 (1999).

49. C. E. Lee et al., "Luminance enhancement of flip-chip light-emitting diodes by geometric sapphire shaping structure," IEEE Photonics Technol. Lett. 20(1-4), 184-186 (2008).

50. X. H. Wang, P. T. Lai, and H. W. Choi, "Laser micromachining of optical microstructures with inclined sidewall profile," J. Vac. Sci. Technol. B 27(3), 1048-1052 (2009).

51. B. Sun et al., "Shape designing for light extraction enhancement bulk-GaN light-emitting diodes," J. Appl. Phys. 113(24), 243104 (2013).

52. S.-J. Chang et al., "GaN-based light-emitting diodes prepared with shifted laser stealth dicing," J. Disp. Technol. 12(2), 195-199 (2015).

53. Y. Guo et al., "Sapphire substrate sidewall shaping of deep ultraviolet light-emitting diodes by picosecond laser multiple scribing," Appl. Phys. Express 10(6), 062101 (2017).

54. J. Yun and H. Hirayama, "Investigation of the light-extraction efficiency in $280 \mathrm{~nm} \mathrm{AlGaN}$ based light-emitting diodes having a highly transparent p-AlGaN layer," J. Appl. Phys. 121(1), 013105 (2017).

55. L. Zhang et al., "High-quality AlN epitaxy on nano-patterned sapphire substrates prepared by nano-imprint lithography," Sci. Rep. 6, 35934 (2016).

56. D. Lee et al., "Improved performance of AlGaN-based deep ultraviolet light-emitting diodes with nano-patterned AlN/sapphire substrates," Appl. Phys. Lett. 110(19), 191103 (2017).

57. Y. Zhang et al., "Near milliwatt power AlGaN-based ultraviolet light emitting diodes based on lateral epitaxial overgrowth of AIN on Si(111)," Appl. Phys. Lett. 102(1), 011106 (2013).

58. H. Y. Ryu, "Large enhancement of light extraction efficiency in AlGaN-based nanorod ultraviolet light-emitting diode structures," Nanoscale Res. Lett. 9(1), 58 (2014).

59. M. Djavid and Z. Mi, "Enhancing the light extraction efficiency of AlGaN deep ultraviolet light emitting diodes by using nanowire structures," Appl. Phys. Lett. 108(5), 051102 (2016).

60. M. Djavid et al., "Effects of optical absorption in deep ultraviolet nanowire light-emitting diodes," Photonics Nanostruct. Fundam. Appl. 28, 106-110 (2018).

61. M. S. Alias et al., "Enhancing the light-extraction efficiency of an AlGaN nanowire ultraviolet light-emitting diode by using nitride/air distributed Bragg reflector nanogratings," IEEE Photonics J. 9(5), 1-8 (2017).

62. X. Liu et al., "Selective area epitaxy of AlGaN nanowire arrays across nearly the entire compositional range for deep ultraviolet photonics," Opt. Express 25(24), 30494-30502 (2017).

63. Q. Wang et al., "Highly efficient, spectrally pure $340 \mathrm{~nm}$ ultraviolet emission from $\mathrm{Al}_{\mathrm{x}} \mathrm{Ga}_{1-\mathrm{x}} \mathrm{N}$ nanowire based light emitting diodes," Nanotechnology 24(34), 345201 (2013).

64. P.-M. Coulon et al., "Hybrid top-down/bottom-up fabrication of regular arrays of AlN nanorods for deep-UV core-shell LEDs," Phys. Status Solidi B 255, 1700445 (2018).

65. J. Dai et al., "Fabrication of AlGaN nanorods with different Al compositions for emission enhancement in UV range," Nanotechnology 28(38), 385205 (2017).

Yanan Guo is an assistant professor at the Institute of Semiconductors, Chinese Academy of Sciences. She received her BS degree in physics from Xiamen University in 2012, and her PhD in materials physics and chemistry from the University of Chinese Academy of Sciences in 2017. Since then, she joined the Semiconductor Lighting R\&D Center of CAS to continue the research. 
Her current research interests are mainly focused on the performance enhancement of AlGaNbased ultraviolet emitters.

Jianchang Yan, $\mathrm{PhD}$, is a professor at the Institute of Semiconductors, Chinese Academy of Sciences. He received his bachelor's degree from Tsinghua University, and his $\mathrm{PhD}$ in microelectronics and solid-state electronics from the Graduate University of Chinese Academy of Sciences. In 2009, he joined the Semiconductor Lighting R\&D Center of CAS to continue the research work on III-nitride material and devices. He was visiting scholar of Paris Sud University in 2015 to 2016 . He is the leader of the III-nitride ultraviolet light-emitting device group, focusing on the research of $\mathrm{Al}(\mathrm{Ga}) \mathrm{N}$-based UV LEDs and UV LDs.

Biographies for the other authors are not available. 